\title{
A new endoscopic closure method for gastric mucosal defects: feasibility of endoscopic hand suturing in an ex vivo porcine model (with video)
}

Authors

Institutions
Osamu Goto' ${ }^{1}$, Motoki Sasaki ${ }^{1}$, Hiroyuki Ishii ${ }^{1}$, Joichiro Horii', Toshio Uraoka', Hiroya Takeuchi' ${ }^{2}$, Yuko Kitagawa $^{2}$, Naohisa Yahagi ${ }^{1}$

${ }^{1}$ Division of Research and Development for Minimally Invasive Treatment, Cancer Center, Keio University School of Medicine ${ }^{2}$ Department of Surgery, Keio University School of Medicine, Tokyo, Japan submitted 4. March 2014 accepted 17. March 2014

\section{Bibliography}

DOI http://dx.doi.org/

10.1055/s-0034-1377180

Published online: 23.6.2014

Endoscopy International Open

2014; 2: E111-E116

(c) Georg Thieme Verlag KG

Stuttgart · New York

E-ISSN 2196-9736

\section{Corresponding author}

Osamu Goto, MD, PhD

Division of Research and Development for Minimally Invasive Treatment, Cancer Center

Keio University School of Medicine

35 Shinanomachi, Shinjuku-ku Tokyo 160-8582

Japan

Fax: +81-3-5363-3895

ogotou-gi@a3.keio.jp
Background and study aims: More secure endoscopic closure techniques for iatrogenic gastric defects are required for safe endoscopic surgery. We developed a novel endoscopic suturing method, endoscopic hand suturing (EHS), of gastric mucosal defects and determined its feasibility and efficacy ex vivo.

Materials and methods: We created 24 mucosal defects (each $2 \mathrm{~cm}$ in diameter) by endoscopic submucosal dissection. The following three techniques were tested: EHS with a 3-0 barbed suture that was grasped with biopsy forceps $(n=6)$ or a prototype through-the-scope needle holder $(n=6)$ by endoscopy, looping with endoloops $(n=$ 6) by endoscopy, and clipping with hemoclips ( $\mathrm{n}=$ 6) by hand. The mucosal edges were attached to each other at three points. The closure strength was compared among the three groups, and the

\section{Introduction \\ $\nabla$}

Endoscopic submucosal dissection (ESD) is accepted, particularly in Asian countries, as a minimally invasive endoscopic approach to curing early stage cancer without organ resection [1-5]. Delayed bleeding is a major complication after gastric ESD that occurs in approximately $5 \%$ of patients [6-8]. Delayed perforation can also develop after successful resection [9]. Although the definitive causes of these complications are unknown, the exposure of an iatrogenic mucosal defect to gastric acid may be an important factor. When such defects are created in patients at risk for complications, closure of the defect with hemoclips or a detachable snare is sometimes attempted [10-12].

Suturing with a needle and suture thread can yield a secure closure when the alimentary tract is surgically anastomosed. If it is possible to firmly close an iatrogenic defect by suturing the mucosa endoscopically, delayed bleeding after ESD or anastomotic leakage after endoscopic translum- procedural duration was compared between the EHS and looping groups.

Results: All 12 lesions were completely closed by EHS. The median strength of the closure, measured with a spring scale, was significantly greater in the EHS group $(0.74 \mathrm{~kg})$ than in the looping group $(0.33 \mathrm{~kg}, P=0.0012)$ or clipping group $(0.07 \mathrm{~kg}, P=0.0009)$. The median procedural duration did not significantly differ between the EHS and looping groups (19.7 vs. 19.8 minutes, $P=$ $1.0000)$. The use of the needle holder significantly reduced the procedural duration compared with the biopsy forceps.

Conclusion: Mucosal defects can be firmly closed with EHS, which may be helpful for establishing a safer and more secure endoscopic surgery.

inal surgery can be more effectively prevented. Therefore, we designed an endoscopic continuous suturing method, endoscopic hand suturing (EHS), in which a needle and suture are used to optimally close iatrogenic mucosal defects. Here, we investigated the feasibility of this method and compared its performance with that of conventional looping and clipping in a porcine model ex vivo.

\section{Materials and methods \\ $\nabla$ \\ Preparation}

We obtained 10 isolated stomachs from pigs that had been previously slaughtered for food. A single endoscopist created 24 mucosal defects (each $2 \mathrm{~cm}$ in diameter) by ESD in these stomachs, which were then placed on an ESD training unit after the insides had been rinsed several times with tap water. Several dots were circumferentially marked by placing 2 -cm plastic disks on the mucosal surface. Normal saline with a small 


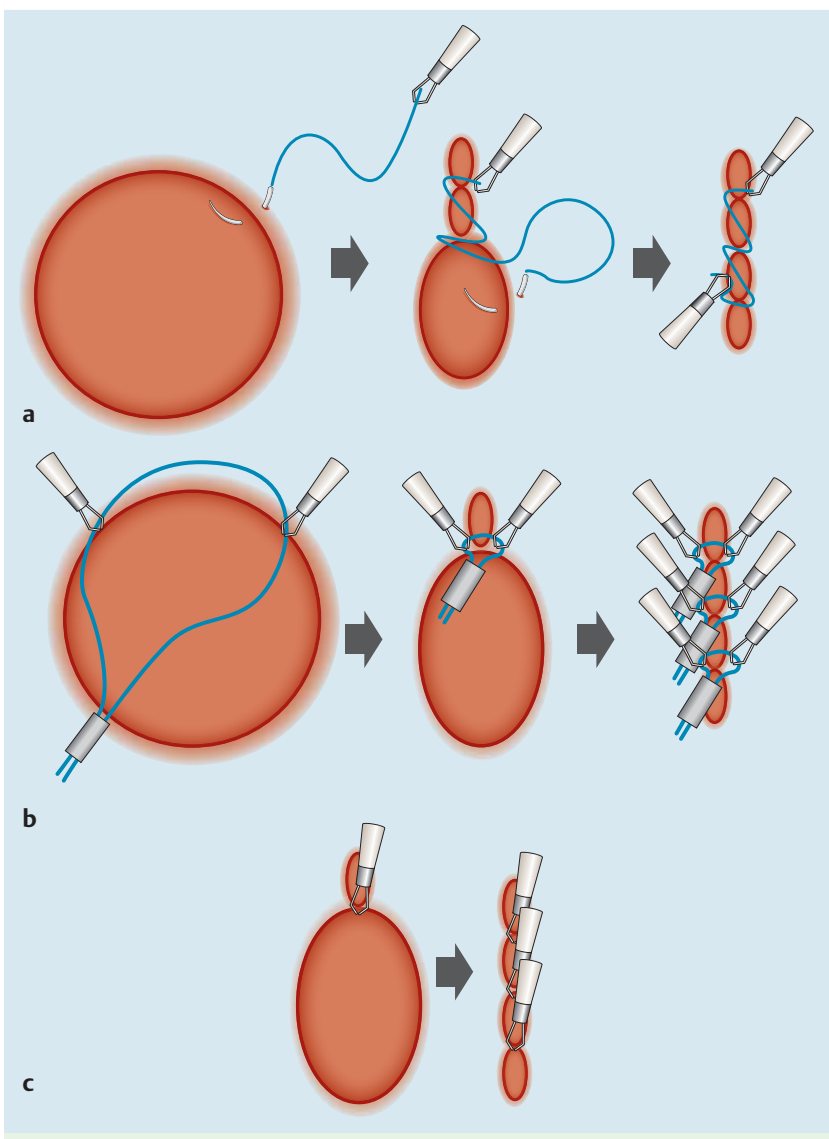

Fig. 1 Comparison of three mucosal lesion closure methods. All mucosal defects were closed at three points. a Endoscopic hand suturing. The defect was continuously sutured endoscopically by using a barbed suture with hemoclips at the end. $\mathbf{b}$ Looping. Endoloops were attached to mucosal edges with hemoclips and closed endoscopically. c Clipping. Mucosal edges were affixed to each other with hemoclips by hand. amount of indigo carmine was injected to create a submucosal cushion. A GIF-2TQ260 M multibending scope with two working channels (Olympus Medical Systems Corp., Tokyo, Japan) was used for ESD and endoscopic closure. The closure methods described below were then applied by one endoscopist and one assistant $(\bullet$ Fig. 1$)$.

\section{Endoscopic hand suturing}

Defects were closed with 15-cm, 3-0 absorbable barbed V-Loc 180 (VLOCL0604) sutures (Covidien, Mansfield, MA, USA) ( Fig.2a). This type of unidirectional, knotless suture was originally developed to facilitate skin suturing, and it has subsequently been applied to gastrointestinal surgeries [13-15]. The short barbs are oriented along the suture to face away from the needle, preventing the suture from sliding backward after the tissues have been tightened. A loop on the tail and the continuous small part of the suture without barbs was cut and anchored with an EZ clip (HX-610 - 90; Olympus). In closing six of the lesions, the needle was grasped with biopsy forceps (FB-220U; Olympus). A 10mm SD-210L-10 electrocautery snare (Olympus) was inserted into a separate working channel to tightly grasp the neck of the biopsy forceps for stabilization ( $\checkmark$ Fig.2b). For the next six lesions, a prototype through-the-scope needle holder (Olympus) was used ( Fig. 2 c-e) $[16,17]$. This device has one swinging lateral jaw and one fixed straight jaw, both of which firmly close when the handle slider is grasped. A button on the handle unlocks and opens the jaws. Irrespective of the shape of the endoscope, the tip of the device can simultaneously rotate as the handle rotates, which accelerates suturing, even inside the stomach. To insert the needle and string gently through the overtube, we grasped the tail of the needle and the suture near the anchor clip with biopsy forceps. We grasped the tail of the needle with the needle holder and placed the anchor clip into another working channel in a retrograde manner. The tip of the needle was or-
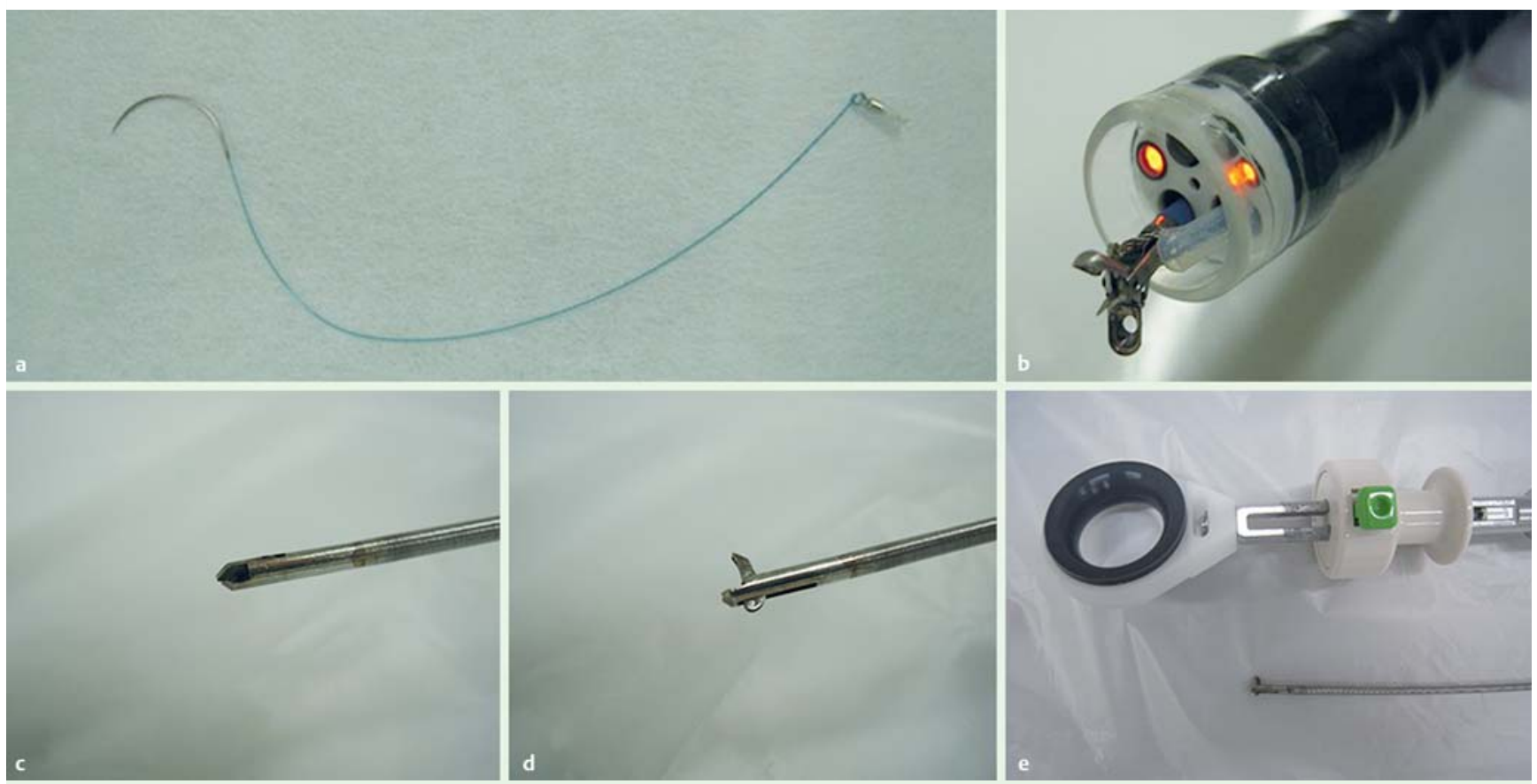

Fig. 2 Tools used for endoscopic hand suturing. a A barbed suture (V-Loc 180) with a hemoclip anchor at its end. b Biopsy forceps fixed with a 10-mm electrocautery snare. c, $\mathbf{d}$ The tip of the prototype through-the-scope needle holder. e The grip of the prototype needle holder. The jaws were tightly closed by gripping the slider, and the button unlocked and opened the jaws. 

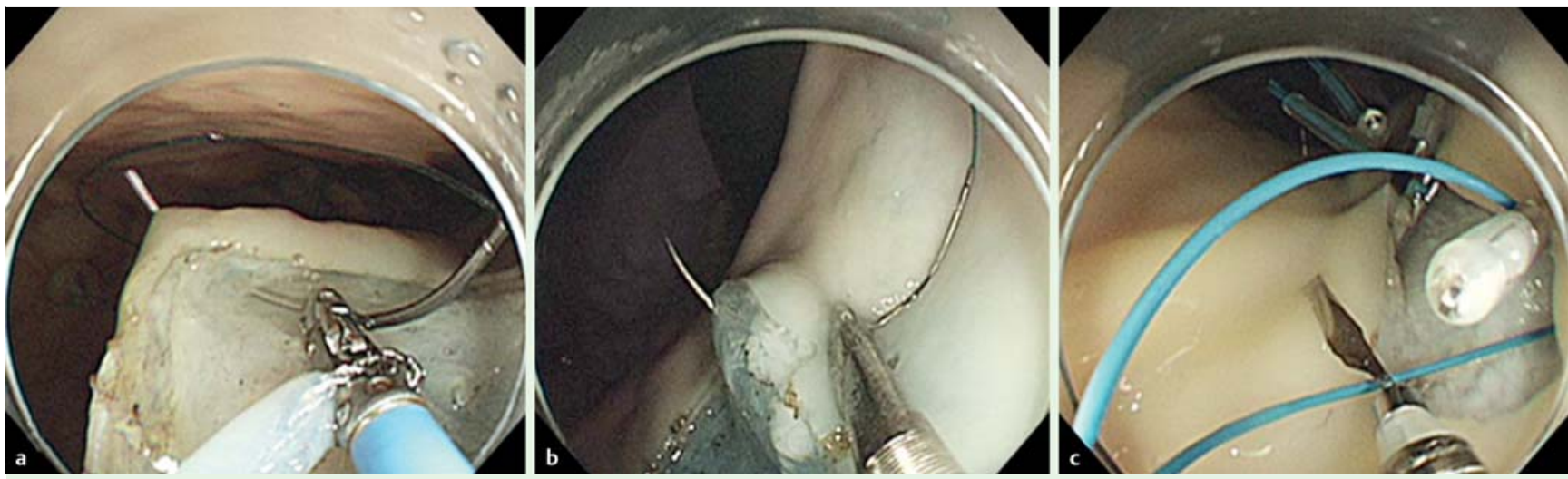

Fig. 3 Endoscopic images of the closure methods. a Endoscopic hand suturing with biopsy forceps. b Endoscopic hand suturing with a prototype needle holder. c Looping.

iented toward the endoscope to avoid potential injury to the mucosa. The needle was released in the stomach and grasped again, and then the mucosal edges and part of the submucosa were continuously sutured to each other longitudinally, from the distal to the proximal sides of the endoscope. After some amount of suturing, the slack part of the suture was tightened by directly grabbing and pulling it with biopsy forceps or the needle holder. After three sutures had been made, one mucosal edge slightly distal to the third suturing point was sutured as a lock. The excess suture and the needle were cut away with a loop cutter (FS-5L-1; Olympus), the end of the suture was clipped to the mucosa, and the needle was removed through the overtube by grasping the tip of the needle with biopsy forceps or the needle holder ( $\bullet$ Fig. 1 a). - Video 1 shows EHS with the needle holder.

\section{Looping}

An MAJ-340 endoloop, $20 \mathrm{~mm}$ in diameter (Olympus), was opened above a mucosal defect and placed at each mucosal edge with an EZ clip (HX-610 - 90 or HX-610-90L; Olympus) [11,12]. Thereafter, the loop was slowly closed by heading a locking unit. The loop was firmly closed and detached. The excess loop was cut and removed with a loop cutter. We spaced three loops along the line to divide the closure seam into four equidistant segments (๑ig. 1b).

\section{Clipping}

We applied three HX-610 - 90 or HX-610 - 90 L EZ clips per lesion. To stitch the mucosal edges to each other without slippage, the procedure was performed by hand after the stomach had been opened as described below. Clips were spaced along the line to divide the closure seam into four equidistant segments ( $\bullet$ Fig. 1 c). We closed six experimentally created mucosal defects under each condition (EHS with biopsy forceps, EHS with the needle holder, looping, and clipping) ( Fig. 3, $\bullet$ Fig.4a-c). We created two artificial lesions in the gastric corpus of nine stomachs for both the EHS and looping groups. For a forward approach, one lesion was located at the posterior wall, and for a retroflex approach, one le- sion was positioned at the lesser curvature. An additional six lesions in total were created for the clipping group by using the remainder of the two stomachs and one additional whole stomach. Endoscopic closure of the lesion on the lesser curvature was initially achieved by a retroflex approach and repeated again on the posterior wall to avoid potential mechanical injury to the sutured lesion on the posterior wall while the endoscope was handled in a retroflex approach. After closure by endoscopy, the stomach was removed from the unit, cut along the greater curvature, and opened with the mucosal surface facing upward to assess the strength of closure. The clipping method was performed on two of the opened stomachs. We also directly cut one additional stomach and opened it for the clipping method after the creation of four mucosal defects.

\section{Measuring outcomes and statistical parameters}

The primary outcome measured in the three groups was the strength of closure, which was assessed with a digital spring scale ( $\bullet$ Fig. $4 \mathrm{~d}$ ). The scale was attached to an 18-gauge needle that was sewn parallel to the suture line on one side of the mucosa and then pulled to the opposite side of the suture line while the other side of the mucosa was held by hand ( Fig.4e). The weight (in kilograms) indicated on the monitor when the closure collapsed was taken as a surrogate marker of closure strength.

The secondary outcome measured was the procedural duration, which we defined as the time elapsed from the first insertion of the needle to the placement of a clip at the end of the suture in the EHS group, and from the first clipping step to place the first loop at the mucosal edge to cutting the excess of the third loop in the looping group.Differences in closure strength and procedural duration between the grasping devices used (biopsy forceps or needle holder) and the approach used (forward or retroflex) were also analyzed in the EHS group.

Strength and procedural duration were analyzed with the Mann-Whitney $U$ test. Statistical significance was set at a $P$ value of $<0.05$. All data were statistically analyzed with JMP version 9.0 software (SAS Institute, Cary, NC, USA).

\section{Video 1}

Endoscopic hand suturing with the prototype needle holder.

online content including video sequences viewable at: www.thieme-connect.de

\section{Results}

$\nabla$

All 24 procedures were completed, including EHS procedures with biopsy forceps ( 6 of $6,100 \%$ ) or the needle holder ( 6 of 6 , $100 \%$ ). We measured the median strength in the three groups ( $\bullet$ Fig. 5 a) and found that closures were significantly tighter in 

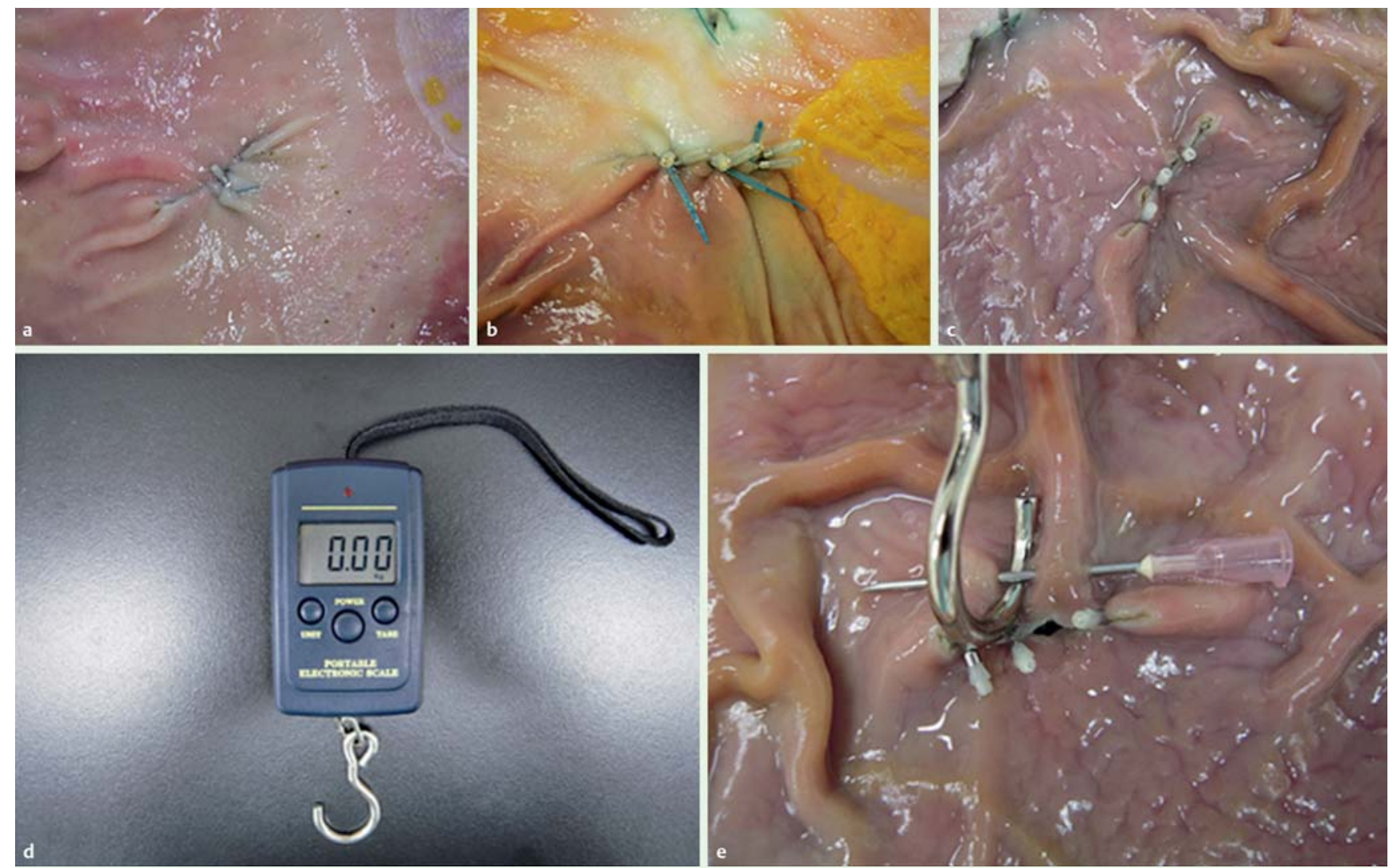

Fig. 4 Closed mucosal defects and measurements. Mucosal defects closed by endoscopic hand suturing (a), looping (b), or clipping (c). d A digital spring scale was used to calculate the strength of the lesion closures at sutured sites. e A digital scale was attached to an 18-gauge needle on one side of the mucosa.

the EHS group $(0.74 \mathrm{~kg}$ [range $0.49-1.16 \mathrm{~kg}$ ]) than in the looping group $(0.33 \mathrm{~kg}$ [range $0.21-0.54 \mathrm{~kg}$ ], $P=0.0012)$ or clipping group $(0.07 \mathrm{~kg}$ [range $0.02-0.31 \mathrm{~kg}], P=0.0009)$. Moreover, the strength was significantly weaker in the clipping group than in the looping group $(P=0.0200)$.

The median procedural duration did not significantly differ between the EHS and looping groups (19.7 minutes [range 12.527.1 minutes] vs. 19.8 minutes [range 15.5-37.4 minutes], $P=$ 1.0000 ) ( Fig.5b). The median procedural duration for stitching in the EHS group (excluding time required for locking, cutting, and clipping the end of the suture) was 13.6 minutes (range 7.9-20.2 minutes), indicating a median time of 4.5 minutes (range 2.6-6.7 minutes) per stitch.

We found no significant difference in median strength between EHS with biopsy forceps or with the needle holder $(0.77 \mathrm{~kg}$ [range $0.63-1.02 \mathrm{~kg}$ ] vs. $0.74 \mathrm{~kg}$ [range $0.49-1.16 \mathrm{~kg}$ ], $P=1.0000$ ), whereas the median procedural duration was significantly shorter with the needle holder than with the biopsy forceps (17.0 minutes [range 12.5-21.2 minutes] vs. 22.7 minutes [range 16.8 27.1 minute], $P=0.0161$ ) ( $\bullet$ Fig. 6 ). The median stitching durations with the biopsy forceps and the needle holder were 16.4 minutes (range 11.3-20.2 minutes) and 10.3 minutes (range 7.9-17.1 minutes), respectively. The time required per stitch tended to be shorter with the needle holder than with biopsy forceps, but the difference was not statistically significant (median 3.4 minutes [range 2.6 - 5.7 minutes] vs. 5.5 minutes [range 3.8 6.7 minutes], $P=0.0538$ ). The median strength and median procedural duration did not significantly differ between the forward and retroflex approaches in the EHS group $(0.72 \mathrm{~kg}$ [range $0.60-$ $1.16 \mathrm{~kg}$ ] vs. $0.79 \mathrm{~kg}$ [range $0.49-1.06 \mathrm{~kg}$ ], $P=0.6884$ and 21.6 minutes [range 12.5-27.0 minutes] vs. 17.5 minutes [range 12.8 - 27.1 minutes], $P=0.6884$, respectively) ( $\bullet$ Fig. 7 ).

\section{Discussion}

$\nabla$

Using an ex vivo porcine model, we found that EHS was feasible and offered firmer closure than conventional looping and clipping methods. Based on our findings, we speculate that EHS would make mucosal defect closure more secure than the other two methods.

Clipping is the most popular method in clinical practice because it is convenient and simple, particularly for patients at a high risk for delayed bleeding due to antiplatelet or antithrombotic medications [10]. However, the findings presented here imply that the mucosal edges attached by clipping are too fragile to stick together for long periods of time. Looping can be used particularly for large lesions that cannot be closed by clipping [11,12], although this method is somewhat tedious and more time-consuming. Here, we found that looping was stronger than clipping, but EHS was more secure and did not take any longer than looping. Therefore, our data suggest that EHS is the most reliable of the three methods tested. Although in practice looping is accompanied by clipping, this combined process is still predicted to be weaker than EHS based on the data presented in this study. Because clipping is far weaker than looping, it would not significantly enhance the strength of looping.

In the looping and clipping groups, closure sites collapsed when the clips became detached as the mucosal edges parted. In contrast, closure sites dehisced when sutures in the EHS group tore 

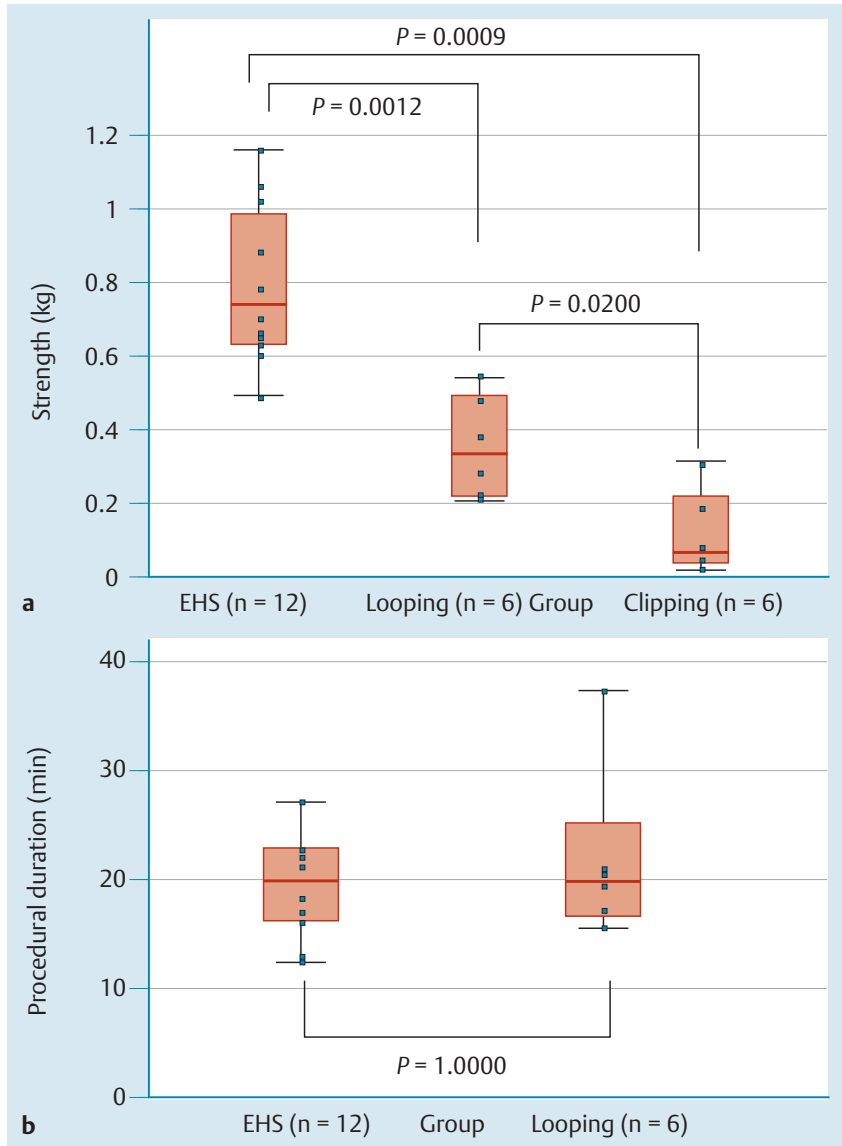

Fig. 5 Comparison of closure strength and procedural duration. a Endoscopic hand suturing is significantly stronger than looping and clipping. b Procedural duration does not significantly differ between the endoscopic hand suturing and looping groups.

the mucosa. This finding suggests that sites closed with EHS will remain intact unless affected by a strong force that is powerful enough to tear the mucosa, whereas mucosal sites closed by looping or clipping may easily dehisce in the presence of less powerful forces, such as food movement or peristalsis.

Many reports have addressed the management of delayed bleeding, and an evidenced-based consensus is gradually forming [6$8,18-20]$; however, no definitive methodology can yet effectively prevent delayed bleeding. Furthermore, delayed perforation can occasionally occur after a successful procedure [9]. The precise cause of delayed perforation is unknown, but excessive thermal injury to the surface of a mucosal defect may be a risk factor. Exposure of a mucosal defect to gastric acid after ESD can also be a risk factor for delayed bleeding and delayed perforation because gastric acid slows the gastric ulcer healing process and stimulates nonbleeding visible vessels or nonperforated thin walls in an iatrogenic mucosal defect. EHS should prevent such complications by preventing the exposure of mucosal defects to gastric acid. Practically, antisecretory medicines, which keep the risk for delayed bleeding low, are used to accelerate the healing of mucosal defects. Therefore, EHS would be more effective in patients who have a high risk for delayed bleeding, such as those who are taking antithrombotic agents, have a coagulopathy or liver cirrhosis, or are being treated for chronic renal failure by hemodialysis.
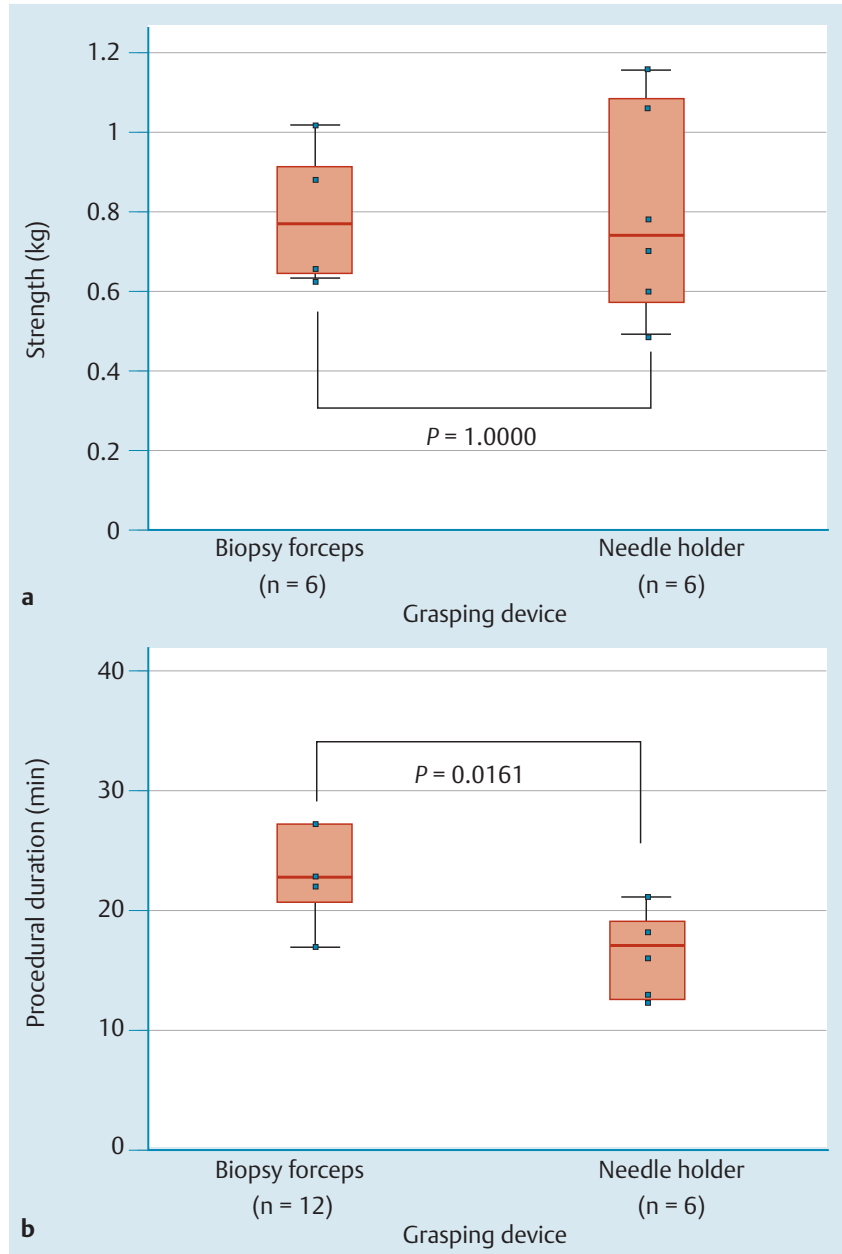

Fig. 6 Comparison of closure strength and procedural duration with different grasping devices. a A prototype needle holder can maintain strength at suture site. $\mathbf{b}$ Procedural duration is significantly shorter when a needle holder is used instead of biopsy forceps.

Over-the-scope clips [21] and the OverStitch Endoscopic Suturing System (Apollo Endosurgery, Austin, TX, USA) [22], which were principally developed to close transmural defects, can also effectively close mucosal defects. A previous study showed comparable strength when wall defects were closed with over-the-scope clips or surgical suturing [23]. The Eagle Claw (Olympus) [24] and Flexible Endostitch (Covidien) [25] are also promising suturing devices for wall closure, but the efficacy of mucosal closure with these devices is unknown, and a comparison with EHS is warranted. On the contrary, EHS may have potential application in the treatment of perforation or anastomosis. Although full-thickness suturing by EHS would be more difficult than mucosal suturing because of collapse of the stomach during the procedure, it might be worth trying in the future.

Our study has some limitations. EHS requires a certain amount of suturing time, a skillful hand for endoscope maneuvering, and some experience with the technique. In addition, extant needles and sutures are not intended for endoscopic suturing of the mucosa. Other limitations of our study are as follows: a relatively small number of lesions closed per technique, the use of hand clipping instead of endoclipping, and the use of porcine stomachs ex vivo. The findings of this study should be considered preliminary at best. Thus, an in vivo porcine trial that included a histologic assessment of healed sutured lesions would be desirable to con- 


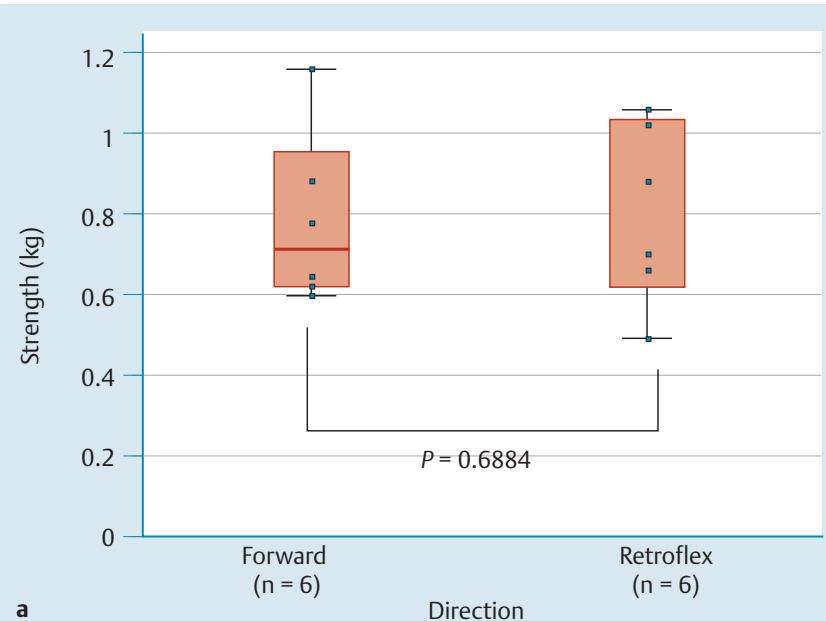

a

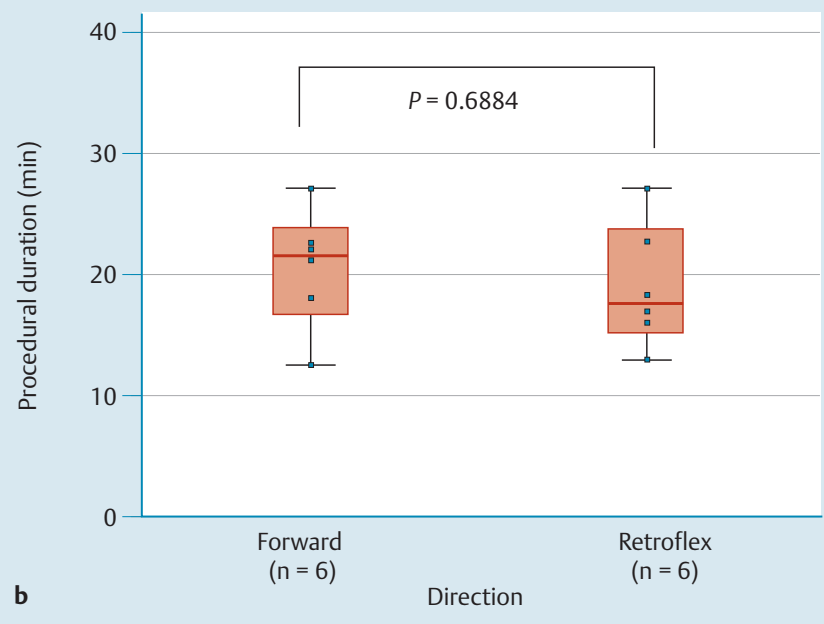

Fig. 7 Comparison of closure strength and procedural duration when the forward or retroflex approach was used. Strength (a) and procedural duration (b) do not significantly differ between the approaches.

firm the technical feasibility and safety of the EHS method. If successful, the porcine trial should be followed by a clinical trial to investigate the clinical benefits of EHS.

In conclusion, we have demonstrated the feasibility and utility of EHS in this ex vivo study. EHS may more effectively prevent complications after gastric ESD than current measures and may be more helpful in establishing a safe and secure endoscopic surgery. A future in vivo animal study and a clinical trial are desired.

Competing interests: The authors have no conflicts of interest or financial ties to disclose. O. G. was funded by a Grant-in-Aid for Young Scientists from the Ministry of Education, Culture, Sports, Science and Technology in Japan in 2011-2012 (Grant No. 23790770).

\section{References}

1 Ono H, Kondo H, Gotoda T et al. Endoscopic mucosal resection for treatment of early gastric cancer. Gut 2001; 48: 225-229

2 Oyama T, Tomori A, Hotta $K$ et al. Endoscopic submucosal dissection of early esophageal cancer. Clin Gastroenterol Hepatol 2005; 3: 67-S70

3 Yahagi N, Uraoka T, Ida Y et al. Endoscopic submucosal dissection using the Flex and the Dual knives. Tech Gastrointest Endosc 2011; 13: 74 78

4 Chung IK, Lee JH, Lee SH et al. Therapeutic outcomes in 1000 cases of endoscopic submucosal dissection for early gastric neoplasms: Korean
ESD Study Group multicenter study. Gastrointest Endosc 2009; 69: $1228-1235$

5 Goto O, Fujishiro M, Kodashima S et al. Outcomes of endoscopic submucosal dissection for early gastric cancer with special reference to validation for curability criteria. Endoscopy 2009; 41: 118-122

6 Goto O, Fujishiro M, Kodashima $S$ et al. A second-look endoscopy after endoscopic submucosal dissection for gastric epithelial neoplasm may be unnecessary: a retrospective analysis of postendoscopic submucosal dissection bleeding. Gastrointest Endosc 2010; 71: 241 - 248

7 Okada K, Yamamoto Y, Kasuga A et al. Risk factors for delayed bleeding after endoscopic submucosal dissection for gastric neoplasm. Surg Endosc 2011; 25: $98-107$

8 Toyokawa T, Inaba T, Omote $S$ et al. Risk factors for perforation and delayed bleeding associated with endoscopic submucosal dissection for early gastric neoplasms: analysis of 1123 lesions. J Gastroenterol Hepatol 2012; 27: 907-912

9 Ikezawa K, Michida T, Iwahashi K et al. Delayed perforation occurring after endoscopic submucosal dissection for early gastric cancer. Gastric Cancer 2012; 15: 111 - 114

10 Choi KD, Jung HY, Lee GH et al. Application of metal hemoclips for closure of endoscopic mucosal resection-induced ulcers of the stomach to prevent delayed bleeding. Surg Endosc 2008; 22: 1882 - 1886

11 Endo $M$, Inomata $M$, Terui $T$ et al. New endoscopic technique to close large mucosal defects after endoscopic mucosal resection in patients with gastric mucosal tumors. Dig Endosc 2004; 16: 372 - 375

12 Lee BI, Kim BW, Kim HK et al. Routine mucosal closure with a detachable snare and clips after endoscopic submucosal dissection for gastric epithelial neoplasms: a randomized controlled trial. Gut Liver 2011; 5: 454-459

13 Zaruby J, Gingras K, Taylor J et al. An in vivo comparison of barbed suture devices and conventional monofilament sutures for cosmetic skin closure: biomechanical wound strength and histology. Anesthet Surg J 2011; 31: $232-240$

14 Demyttenaere SV, Nau P, Henn $M$ et al. Barbed suture for gastrointestinal closure: a randomized control trial. Surg Innov 2009; 16: 237-242

15 Patri P, Beran C, Stjepanovic J et al. V-Loc, a new wound closure device for peritoneal closure - is it safe? A comparative study of different peritoneal closure systems Surg Innov 2011; 18: 145-149

16 Olsina-Kissler JJ, Balsells-Valls J, Dot-Bach J et al. Natural orifice transluminal endoscopic surgery (NOTES): initial experimental results. Cir Esp 2009; 85: 298 - 306

17 Maratka Z, Armengol-Miro JR. Endoscopic diagnosis and therapy in gastroenterology. Bad Homburg: Normed Verlag; 2012

18 Uedo $N$, Takeuchi Y, Yamada $T$ et al. Effect of a proton pump inhibitor or an $\mathrm{H} 2$-receptor antagonist on prevention of bleeding from ulcer after endoscopic submucosal dissection of early gastric cancer: a prospective randomized controlled trial. Am J Gastroenterol 2007; 102: $1610-1616$

19 Takizawa K, Oda I, Gotoda T et al. Routine coagulation of visible vessels may prevent delayed bleeding after endoscopic submucosal dissection - an analysis of risk factors. Endoscopy 2008; 40: 179-183

20 Goto 0 , Fujishiro M, Oda I et al. A multicenter survey of the management after gastric endoscopic submucosal dissection related to postoperative bleeding. Dig Dis Sci 2012; 57: 435-439

21 Schurr MO, Hartmann C, Ho CN et al. An over-the-scope clip (OTSC) system for closure of iatrogenic colon perforations: results of an experimental survival study in pigs. Endoscopy 2008; 40: 584-588

22 Moran EA, Gostout CJ, Bingener J. Preliminary performance of a flexible cap and catheter-based endoscopic suturing system. Gastrointest Endosc 2009; 69: 1375-1383

23 von Renteln D, Schmidt A, Vassiliou MC et al. Natural orifice transluminal endoscopic surgery gastrotomy closure with an over-the-endoscope clip: a randomized, controlled porcine study (with videos). Gastrointest Endosc 2009; 70: 732 -739

24 Pham BV, Raju GS, Ahmed I et al. Immediate endoscopic closure of colon perforation by using a prototype endoscopic suturing device: feasibility and outcome in a porcine model (with video). Gastrointest Endosc 2006; 64: $113-119$

25 Voermans RP, Worm AM, van Berge Henegouwen MI et al. In vitro comparison and evaluation of seven gastric closure modalities for natural orifice transluminal endoscopic surgery (NOTES). Endoscopy 2008; 40: $595-601$ 